\title{
Tip artifacts of microfabricated force sensors for atomic force microscopy
}

\author{
P. Grütter, W. Zimmermann-Edling, and D. Brodbeck \\ Institut für Physik, Universität Basel, Klingelbergstrasse 82, 4056. Basel, Switzerland
}

(Received 13 January 1992; accepted for publication 25 March 1992)

It is demonstrated that due to inevitable intrinsic imperfections in the microfabrication process of atomic force microscopy (AFM) tips, images of rough surfaces can be totally dominated by tip artifacts. These images reflect the mesoscopic tip shape as concluded from a comparison of AFM and scanning electron microscopy images of the tip and sample. These tip artifacts have been found on a scale of $20-600 \mathrm{~nm}$, showing the necessity of characterizing the tip shape in order to make reliable sample-specific statements.

Since its invention in 1986 (Ref. 1) and the general availability of commercial instruments, atomic force microscopy (AFM) has found many applications both in research and technology. ${ }^{2}$ The crucial component of every force microscope is the force sensor: a sharp tip attached to the end of a cantilever. Microfabricated force sensors are commonly used due to their advantageous properties and the possibility of batch fabrication. ${ }^{3}$ In the following we will experimentally demonstrate that in some cases these tips lead to images dominated by tip artifacts on a nanometer-to-micrometer scale. The origin of these artifacts can be traced to inevitable imperfections in the microfabrication of the tips. Since these imperfections are common, they need to be characterized and compared to the dimensions of structures of interest on the sample, especially if quantitative tip-independent statements are to be made. ${ }^{4}$ Previously, artifacts which are due to the convolution of tip shape and sample structure have been described both experimentally and theoretically for scanning tunneling microscopy (STM) tips. ${ }^{5-9}$

For the following study we used a commercial instrument ${ }^{10}$ with microfabricated $\mathrm{Si}_{3} \mathrm{~N}_{4}$ force sensors. As a test sample, we imaged a polycrystalline diamond film grown by conventional thermally activated chemical vapor deposition. ${ }^{11}$ These diamond films have statistically oriented cubo-octahedral crystals $0.5-5 \mu \mathrm{m}$ in size with numerous sharp tips and very steep faccts. Since these diamond films are fairly conductive, their topography can be characterized by scanning electron microscope (SEM) [shown in Fig. 1(a)] and even STM in air. ${ }^{12}$

When imaged with a particular force sensor, the diamond film revealed a morphology consisting of an oriented array of truncated pyramids [Fig. 1(b)]. The rectangles terminating the pyramids have a side length of about 600 nm. If Fig. 1(b) reflected the true sample morphology, it could be attributed to an epitaxially oriented polycrystalline diamond film with a crystallite size of $600 \mathrm{~nm}$. This of course is wrong as is evident when Fig. 1(b) is compared to the SEM images of the sample shown in Fig. 1(a). The correct interpretation of Fig. 1(b) is evident when characterizing the tip shape. SEM images of the tip [shown in Fig. $1(c)]$ reveal that it was not sharp and pointlike as usually assumed, but rather flat instead and terminated by a rectangle of $600-\mathrm{nm}$ side length. This shape is very similar to the truncatcd pyramidal structures observed on the sample.
The convolution of this tip shape with the true sample morphology can explain the AFM image shown in Fig. $1(b)$. We directly demonstrate this with a simulation of the scan process. First, the true sample morphology is measured with a sharp STM tip, giving an array of values $z_{\text {STM }}=f(x, y)$. A single trace of such a STM image is presented at the bottom of $\Gamma$ ig. 1 (d). The relevant tip geometry and dimensions [shown to scale in Fig. 1(d)] were extracted from SEM images of the tip, giving a second array $z_{\text {tip }}=g(x, y)$. We then simulated the AFM scan process in the following way: for each surface element $(x, y)$ we calculated the smallest distance $z_{\mathrm{AFM}}=z_{\mathrm{tip}}-z_{\mathrm{STM}}$. The resulting AFM simulation strongly resembles Fig. 1(b). One can easily recognize this by comparing a trace of the simulation [shown in the center of Fig. 1(d)] with one extracted from Fig. 1(b) [shown in the top part of Fig. 1(d)]. Both these traces look similar and clearly reflect the inverted profile of the tip. We conclude that the many sharp points and corners on the diamond sample effectively image the tip. This supports the interpretation that AFM images acquired with this tip are dominated by tip artifacts. Practically no statements on the sample morphology can be derived from these images. This simulation also demonstrates the well-known fact that the tip can be considerably larger than the smallest and sharpest depressions observed on an image.

It is somewhat surprising that images can be completely dominated by these imperfect tip shapes even on samples which are not very rough compared to the diamond films presented above. Polyhexamethylene (nylon) on glass has the typical AFM morphology as shown in Fig. 2(a): rather smooth, meandering structures less than 30 $\mathrm{nm}$ high are observable. If the tip of Fig. 1(c) is used, the resulting image again is dominated by the now familiar rectangles [Fig. 2(b)]. Very small bumps and height variations on the otherwise smooth polyhexamethylene meanders effectively image the tip. In contrast to the diamond film, electron-based techniques such as STM or SEM are not readily applicable to determine the true sample morphology of polyhexamethylene due to its insulating nature and the small-scale nature of its morphology. One has to rely on AFM with a tip sharper than the relevant sample dimensions.

The origin of the truncated pyramidal tip shape must be traced to the microfabrication of the force sensors, since similar tip shapes can be found on many other force sen- 


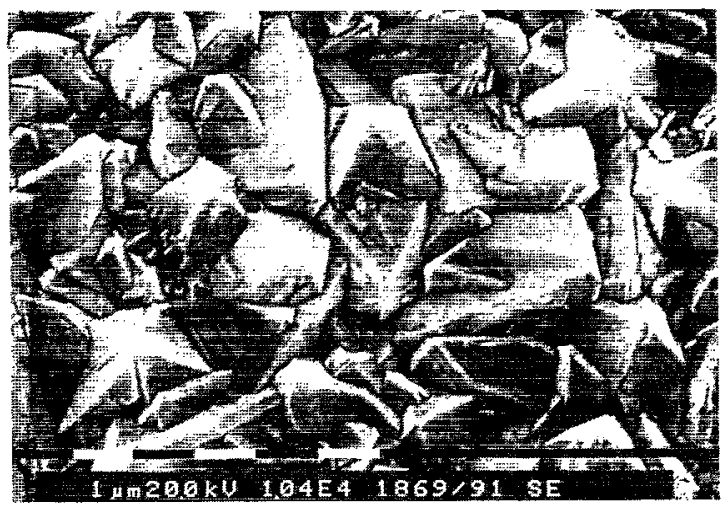

(a)

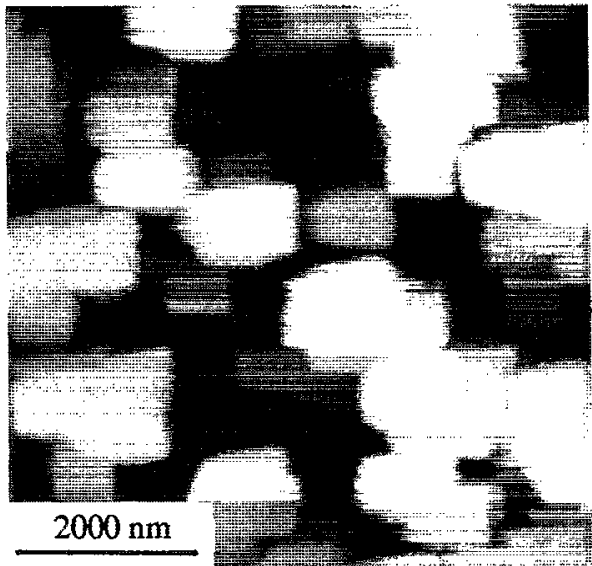

(b)

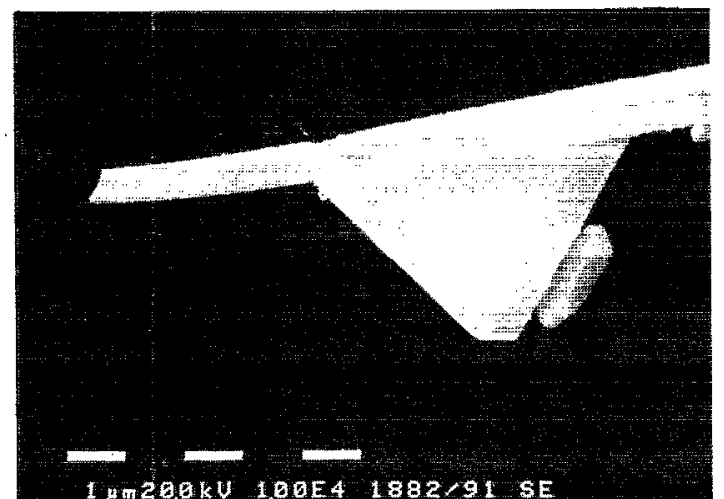

(c)

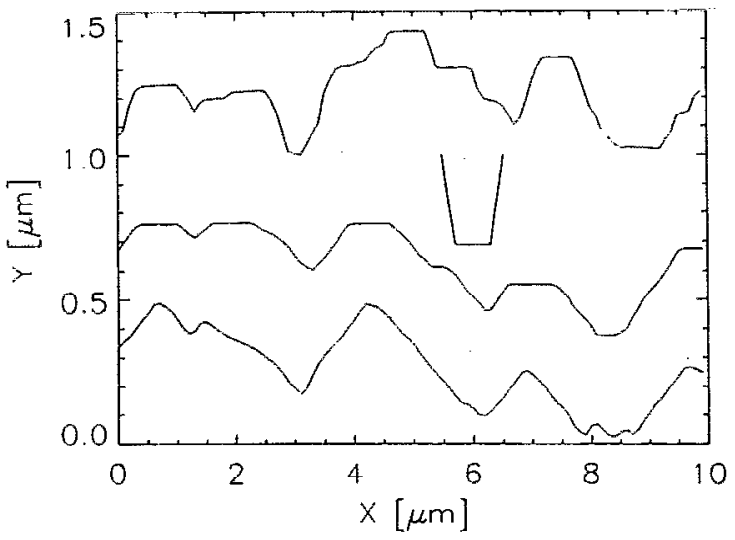

(d)

FIG. 1. (a) SEM of diamond film, (b) AFM image of diamond film, (c) SEM of $\mathrm{Si}_{3} \mathrm{~N}_{4}$ tip used to acquire Figs. 1(b), 2(b) and 3. (d) Convolution of tip and sample structure. Bottom: STM scan line of sample. Middle: Result of numerical simulation of AFM scan with tip geometry taken from (c). The tip shown in the inset looks distorted when compared to (c) due to the different horizontal and vertical scales. Top: Experimental AFM scanline [from (b)] showing tip convolution effect.

sors even prior to their use in the AFM. One of the first steps in producing $\mathrm{Si}_{3} \mathrm{~N}_{4}$ force sensors is to etch a pyramidal pit in the surface of a (100) Si wafer. ${ }^{3}$ This is done by suitably masking a (100) Si wafer and etching a small square opening into the masking film. The exposed $\mathrm{Si}$ is then etched with an anisotropic etchant. The etch process self-terminates when the volume of Si removed is bound by (111) planes, which in an ideal case thus leads to a pointlike termination of the pit. $\mathrm{A} \mathrm{Si}_{3} \mathrm{~N}_{4}$ film is then deposited and conforms the shape of the pyramidal pit. As already pointed out by Albrecht et al., the tip may not terminate exactly in a point if the opening in the masking film is not cxactly square. ${ }^{3}$ This can be a result of mask misalignment, which can even vary locally, e.g., due to the intrinsic roughness of the wafer or a stray dust particle. Furthermore, the etching process might be interrupted before selftermination due to local variations of parameters controlling the etching speed. As a result of all these nonideal processes, it is not surprising that many tips terminate in wedgelike and rectangular shapes. Indeed, we have found rectangles with a side length between 20 and $600 \mathrm{~nm}$ in three out of ten tips tested which were randomly selected from different parts of a wafer. On a given wafer perfect tips can be next to ones with truncated pyramidal tips since the mechanisms leading to imperfect tips can vary locally.
The tip imperfection presented above is on a large enough scale to allow casy visualization by SEM. We have often observed similar tip artifacts on smaller length scales down to $20 \mathrm{~nm}$. This is no surprise considering the inevitable imperfections of the microfabrication process which leads to truncated pyramidal tips. On small length scales, however, the occurrence of a tip artifact in a particular set of images can be less obvious. Furthermore, highresolution tip inspection by SEM is very difficult, one of the reasons being the insulating nature of $\mathrm{Si}_{3} \mathrm{~N}_{4}$, which generally makes a conductive coating necessary.

The mesoscopic tip geometry determines the length scales on which images represent sample characteristics that are free of tip convolution artifacts. ${ }^{4}$ The intrinsic variations of mesoscopic tip dimensions have important consequences for AFM measurements which should be taken into account if the obtained results are to be tip independent and thus comparable. Characterization of AFM tips on a mesoscopic scale is often neglected even when quantitative measurements, such as roughness or friction and adhesion experiments, are performed. Furthermore, even in atomic resolution images the mesoscopic tip shape plays an important role as it determines the attractive part of the total tip-sample interaction. ${ }^{13}$

Clearly, it is necessary to find suitable test and calibra- 

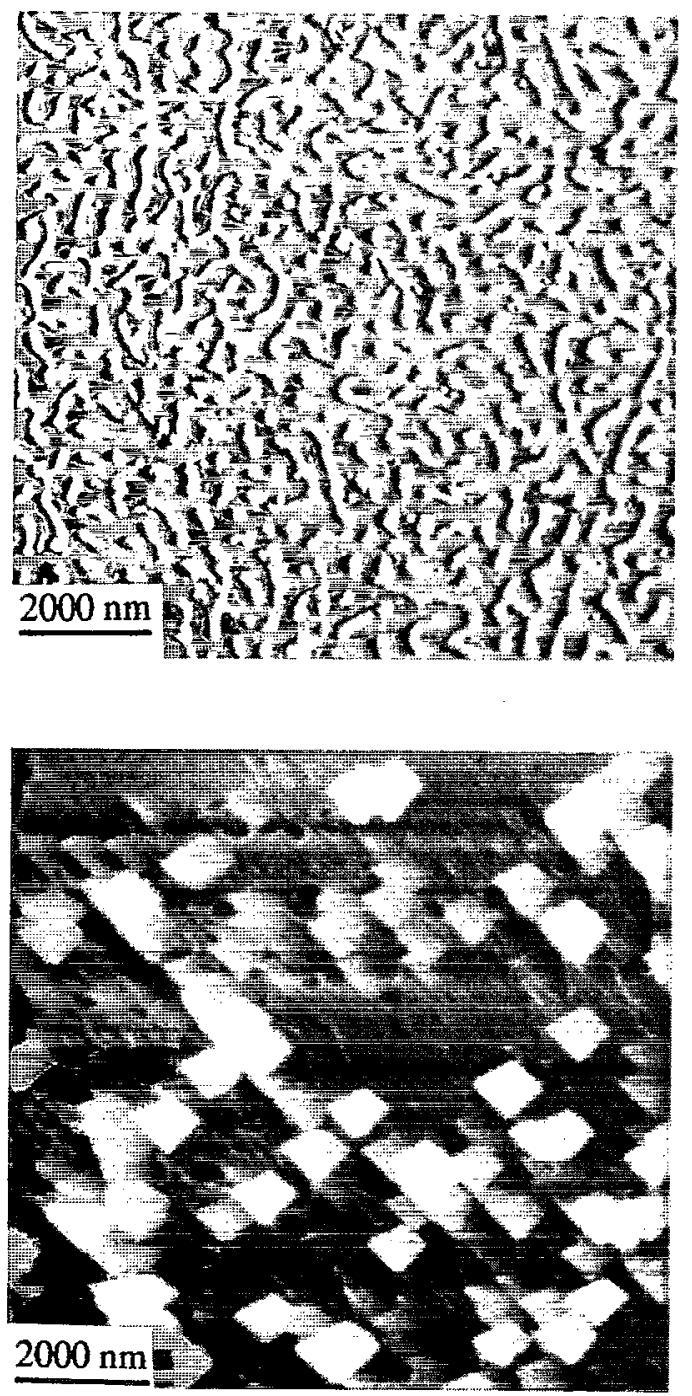

FIG. 2. (a) AFM image of polyhexamethylene (nylon) spin coated onto glass. (b) AFM image of same sample with tip of Fig. 1(c) showing strong tip artifact.

tion samples to determine the mesoscopic tip shape. We would like to emphasize that the very same tip used for Figs. 1(b) and 2(b) is capable of atomic-scale resolution as is demonstrated by imaging highly oriented pyrolytic graphite (Fig. 3): atomic resolution on a flat sample does not charactcrize the mesoscopic tip shape, and a small protrusion on the otherwise flat tip is sufficient for atomicscale contrast. The diamond film used in this study is a suitable test sample due to the many tips, sharp edges, and dimensional variations, allowing a reasonable in situ tip characterization. It would be even more useful to have mesoscopically better defined (e.g., microfabricated) tip calibration samples on a nanometer-to-micrometer scale.

In summary, we have shown that $\mathrm{Si}_{3} \mathrm{~N}_{4}$ microfabri-

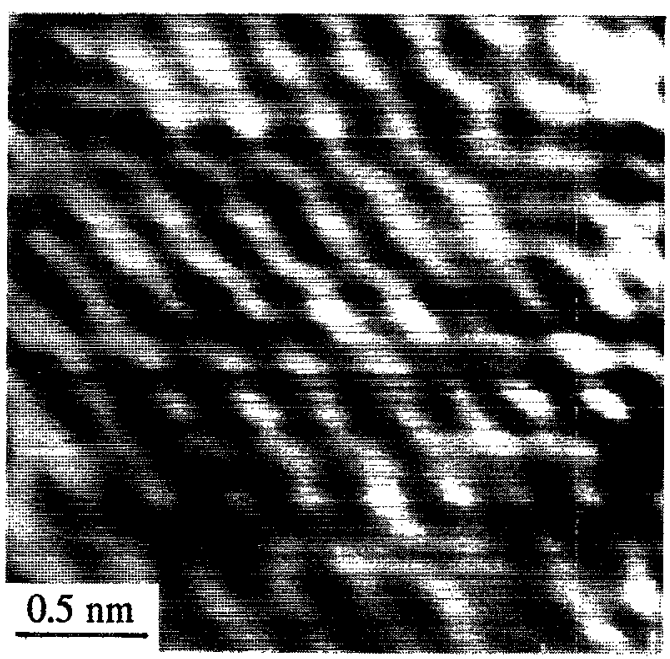

FIG. 3. Atomic-scale resolution by AFM on highly oriented pyrolytic graphite with the tip of Fig. 1(c). This is a slightly Fourier-filtered image acquired at a constant repulsive force of about $10 \mathrm{nN}$.

cated tips can have the shape of truncated pyramids with flat, rectangular ends between 20 and $600 \mathrm{~nm}$ in size. This is a consequence of inevitable imperfections of the microfabrication process. It is essential to know the mesoscopic tip geometry, especially when interpreting AFM images on rough surfaces in order to understand and control the influence of tip convolution.

We would like to thank R. Guggenheim and E. Zuberbuihler for the SEM images. The diamond films were made by $\mathrm{H}$. Sprang. J. Fünfschilling supplied the polyhexamethylene samples. P. G. gratefully acknowledges a stipend by the Treubel Fonds, Basel. This work was supported by the Swiss National Science Foundation and the Kommission zur Förderung der wissenschaftlichen Forschung.

${ }^{1}$ G. Binnig, C. F. Quate, and C. Gerber, Phys. Rev. Lett. 56, 930 (1986). ${ }^{2}$ For recent reviews see, e.g.: D. Rugar and P. Hansma, Phys. Today 43, 23 (1990); D. Sarid and V. Elings, J. Vac. Sci. Technol. B 9, 431 (1991); E. Meyer and J. Frommer, Phys. World 4, 46 (1991).

${ }^{3}$ T. R. Albrecht, S. Akamine, T. E. Carver, and C. F. Quate, J. Vac. Sci. Technol. A 8, 3386 (1990).

${ }^{4}$ M. Stedman and K. Lindsey, SPIE Surf. Meas. Charact. 1009, 56 (1988).

${ }^{5}$ Ph. Niedermann and O. Fischer, J. Microsc. 152, 93 (1988).

${ }^{6}$ G. Reiss, J. Vancea, H. Wittmann, J. Zweck, and H. Hoffmann, J. Appl. Phys. 67, 1156 (1990).

${ }^{7}$ G. Reiss, H. Brückel, J. Vancea, R. Lecheler, and E. Hastreiter, J. Appl. Phys. 70, 523 (1991).

${ }^{8}$ E. J. van Loenen, D. Dijkkamp, A. J. Hoeven, J. M. Lenssinck, and J. Dieleman, Appl. Phys. Lett. 56, 1755 (1990).

'J. P. Pelz and R. H. Kock, Phys. Rev. B 41, 1212 (1990).

${ }^{10}$ Nanoscope II, Digital Instruments Inc., Santa Barbara, CA 93117.

${ }^{11}$ S. Matsumoto, Y. Sato, and M. Tsutsumi, J. Mater. Sci. 17, 3106 (1982).

${ }^{12}$ W. Zimmermann-Edling, H.-G. Busmann, H. Sprang, and I. V. Hertel, Ultramicroscopy 40, 1 (1992).

${ }^{13}$ F. O. Goodman and N. Garcia, Phys. Rev. B 43, 4728 (1991). 